included respiratory distress $(\mathrm{p}=0.000$, risk $=4.006)$, the use of nasogastric tube $(p=0.017, O R=3.281)$ and the use of triple antibiotics $(p=0.001$, risk $=1.432)$. Factors associated with the presence of bloody gastric aspirate included the use of nasogastric tube $(\mathrm{OR}=1.629, \mathrm{p}=0.000)$ and the presence of hemostatic disorders $(\mathrm{OR}=3.143, \mathrm{p}=0.039)$. It was also associated with lower hemoglobin levels $(\mathrm{p}=0.000)$.

Conclusion SRML represents an under-diagnosed problem in NICUs. Absence of bloody gastric aspirate does not exclude stressrelated mucosal lesions.

\section{CORRELATION BETWEEN HELICOBACTER PYLORI SEROLOGIC TESTS WITH RAPID URASE AND HISTOLOGY IN CHILDREN}

doi:10.1136/archdischild-2012-302724.0717

'A Rezaianzadeh, ${ }^{2} \mathrm{MH}$ Imanieh, ${ }^{3} \mathrm{SM}$ Dehghani, ${ }^{3} \mathrm{M}$ Haghighat. 'Epidemiology, Shiraz University of Medical Sciences; ${ }^{2}$ Paediatrics, Shiraz University of Medical Science; ${ }^{3}$ Paediatrics, Shiraz University of Medical Sciences, Shiraz, Iran

Background Helicobacter pylori infection is a common infection that affects the human being. This infection also affects the children. Different diagnostic methods such as serology, stool antigen detection, rapid urease test and histology detect this microorganism. The aim of this study was to determined correlation between serology and histology/rapid urease test.

Methods Two groups were selected and matched for age and sex. Seventy seven children with confirmed $\mathrm{H}$. pylori infection as they had positive rapid urease test and histology concomitantly were compared with 77 healthy children. Both case and control groups checked serologically for detection of anti $\mathrm{H}$. pylori IgM, IgG and IgA antibody titers.

Results Three Cut-off points were 3.3 U/ML for IgA, 6.4 U/ML for IgM, and 9.9 U/ML for IgG. Antibody titers were compared with gold standard methods including histologic and rapid ureas tests. IgA level had a sensitivity of $64 \%$, specificity of $58 \%$, accuracy of $59.3 \%$, positive predictive value of $31.5 \%$, and negative predictive value of $76.9 \%$. IgM level had a sensitivity of $76 \%$, specificity of $36.1 \%$, accuracy of $74.2 \%$, positive predictive value of $31.5 \%$, and negative predictive value of $76.9 \%$. IgG level had a sensitivity of $58.6 \%$, specificity of $61.3 \%$, accuracy of $60.6 \%$, positive predictive value of $36.9 \%$, and negative predictive value of $79.3 \%$.

Conclusion These antibodies have a relatively high negative predictive value and a low positive predictive value. So, their negative results are more valuable. The most sensitive antibody is IgM and most specific antibody is $\operatorname{IgG}$

\section{INFANT FEEDING FOLLOWING GASTROSCHISIS REPAIR}

doi:10.1136/archdischild-2012-302724.0718

A Hickey, C Battersby, P Charlesworth, M O' Meara, J Hind, K Tavener. Paediatrics, Kings College Hospital, London, UK

Background and Aims Establishment of enteral feeding is crucial in gastroschisis. Breastmilk may be protective against early complications. Mothers are counselled by breast feeding advisors in our institution. We reviewed initial and discharge feed in a cohort of simple and complex cases.

Methods Retrospective analysis of feeding outcomes in 46 patients between August 2008 and Jan 2012 in a single centre. Complex cases were defined as those with closed gastroschsisis, bowel atresia, perforation, obstruction or necrosis, stoma or necrotising enterocolitis.

Results Maternal milk (MEBM) was initiated in 42 (91\%) infants. MEBM and donor breast milk for $2(4.5 \%)$ and specialised formula(SF) for $2(4.5 \%)$.
Abstract 718 Table 1 Discharge Feed

\begin{tabular}{lcc}
\hline DISCHARGE FEED & SIMPLE(SG) $\mathbf{n}=\mathbf{3 1}$ & COMPLEX(CG) $\mathbf{n = 1 5}$ \\
\hline MBM & $16(52 \%)$ & $1(7 \%)$ \\
MBM/SF & $3(10 \%)$ & $2(13 \%)$ \\
MBM/TF & $5(16 \%)$ & 0 \\
SPECIALISED FEED(SF) & $2(6 \%)$ & $12(80 \%)$ \\
TERM FORMULA(TF) & $5(16 \%)$ & 0 \\
\hline
\end{tabular}

Discharge feed was all or part breast milk in $78 \%$ of the SG group versus $20 \%$ in the CG, $p=0.0001$. It was breast milk exclusively in $52 \%$ of the SG group versus $7 \%$ of the CG, $p=0.0025$. Median days to full feeds were 16 in the SG versus 67 in the CG, $p<0.0001$.

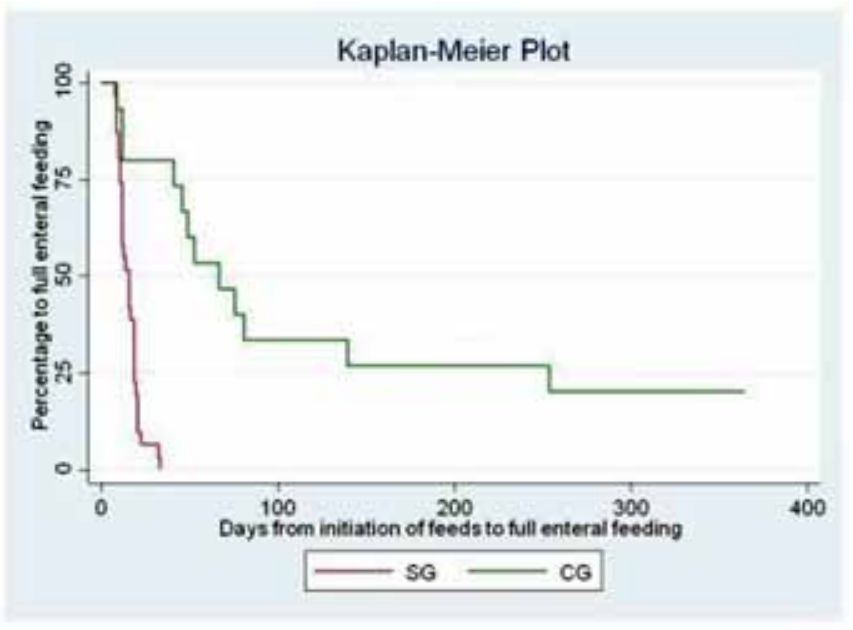

Abstract 718 Figure 1 Time

Conclusions CG cases were less likely to be receiving any breast milk on discharge. This has implications for medical and parental expectations of potential outcome.

\section{THE EFFECT OF A FISH-OIL-BASED LIPID EMULSION ON THE PARENTERAL NUTRITION-ASSOCIATED LIVER DISEASE IN VERY LOW BIRTH WEIGHT INFANTS}

doi:10.1136/archdischild-2012-302724.0719

M Chang, H Kang. Department of Pediatrics, Chungnam National University Hospital, Daejeon, Republic of Korea

Background and Aims Prolonged parenteral nutrition (PN) can cause parenteral nutrition-associated liver disease (PNALD) in very low birth weight infants (VLBWIs). Evidence has suggested soybean-oil-based lipid emulsion (SOLE) is a contributing factor to the development of PNALD. In this study, we investigated the effect of fish-oil-based lipid emulsion (FOLE) on incidence and severity of PNALD in VLBWIs compared with SOLE.

Methods Retrospective review of 66 VLBWIs who received PN for more than 14 days in our NICU from January 2007 to December 2010 was performed. Patients were divided into two groups: SOLE Group ( $n=30$, January 2007-March 2009), received Intralipid $\AA$ (Choongwae Pharma Corporation, Seoul, Korea) and FOLE group ( $\mathrm{n}=36$, June 2009-December 2010), received SMOFlipid $®$ (Fresenius Kabi AG, Bad Homburg, Germany). The Clinical and laboratory findings were evaluated.

Results There were no significant differences in the demographic features and major morbidities between two groups. The peak level of serum direct bilirubin were markedly lower in the FOLE group compared with the SOLE group $(2.21 \pm 2.16$ vs. $3.16 \pm 2.20 \mathrm{mg} / \mathrm{dL}$, 
$p=0.011)$. And the peak level of AST (68.72 \pm 68.00 vs. $106.67 \pm 79.09$ $\mathrm{IU} / \mathrm{L}, p=0.008)$ and ALT (30.92 \pm 29.72 vs. $53.70 \pm 40.86 \mathrm{IU} / \mathrm{L}, p=0.002)$ were significant lower in the FOLE group compared with the FOLE group. However, the peak levels of serum triglyceride were similar. $(245.22 \pm 128.47$ vs. $226.97 \pm 99.49 \mathrm{mg} / \mathrm{dL}, p=0.747)$.

Conclusions The use of FOLE in VLBW infants may reduce the risk of PNALD.

\section{ADEQUATE POSTNATAL DIAGNOSTIC MODALITIES FOR PRENATALLY DIAGNOSED BILIARY CYSTIC MALFORMATIONS}

doi:10.1136/archdischild-2012-302724.0720

T Okada, S Honda, H Miyagi, A Taketomi. Department of Gastroenterological Surgery I, Hokkaido University Graduate School of Medicine, Sapporo, Japan

Background and Aims The aim of this study was to determine an appropriate postnatal diagnostic management plan for prenatally diagnosed biliary cystic malformation (BCM) patients.

Methods From 2002 to 2011, a total of 27 consecutive children with pancreaticobiliary maljunction (PBM) were treated at our institute. Eight (29.6\%) of our 27 patients with choledochal cyst (CC) were diagnosed prenatally and examined clinically. Prenatally diagnosed cystic biliary atresia (BA) was noted in 2 patients with type 1 cystic BA. The clinical data, preoperative imaging findings, and final diagnosis using intraoperative cholangiography were evaluated in these BCM patients.

Results Infants with prenatally diagnosed CC were divided into two groups after birth: a symptomatic group of 5 patients, and an asymptomatic group of 3 patients. According to CC patients, ultrasonography (US) did not reveal a PBM in all 8 CC patients, although the main pancreatic duct was shown in 2 CC patients. The PBM and main pancreatic duct were shown by MRCP at high rates of 80 and $60 \%$, respectively, compared with US and dynamic CT. In cystic BA patients, US and MRCP showed that the gallbladder was atrophic in both of the two cystic BA patients compared with the CC patients. There was not triangular cord sign in the two by US.

Conclusion This study clearly showed that, in some cases, such as prenatally diagnosed BCM, MRCP eliminates the need for endoscopic retrograde cholangiopancreatography (ERCP) because of its excellent sensitivity and specificity, thus avoiding an invasive procedure with marked radiation exposure.

\section{THE EFFECT OF THE RED WINE POLYPHENOL RESVERATROL ON CHOLESTASIS: ANTI-APOPTOTIC, MITOCHONDRIAL BIOGENESIS AND AUTOPHAGY}

doi:10.1136/archdischild-2012-302724.0721

'MM Tiao, ${ }^{2} \mathrm{~F}$ Huang, Mitochondrial Group. 'Pediatrics, Kaohsiung Chang Gung Memorial Hospital and Chang Gung University College of Medicine; 'Pediatrics, Kaohsiung Veterans General Hospital, Kaohsiung, Taiwan R.O.C.

Background and Aims Mitochondria are known to be involved in cholestatic liver injury. The potential protective effect of resveratrol in cholestatic liver injury and the possible roles of autophagy and apoptosis induction in this process are not yet clear. The aim of this study is to determine whether resveratrol administration after bile duct ligation can reduce cholestasis-induced liver injury through modulating apoptosis, mitochondrial biogenesis and autophagy.

Methods A rat model of cholestasis was established by bile duct ligation (BDL) and compared with a sham group receiving laparotomy without BDL, with resveratrol or control treatments following BDL. The expression of proteins involved in the apoptotic and autophagic pathways were analyzed by western blotting. Apoptosis was examined by TUNEL staining.

Results In the resveratrol/BDL group LC3-II upregulation persisted for 1-7 days, Bax was downregulated and catalase was upregulated at 3-7 days after resveratrol treatment. The decline in mitochondrial DNA copy number was reversed at 3-7 days. Caspase 3 expression was significantly downregulated at 3-7 days in the resveratrol group. TUNEL staining showed significant numbers of apoptotic liver cells appeared in livers 3-7 days after BDL and that was decreased by resveratrol treatment.

Conclusions Our results indicate that early resveratrol treatment reverses impaired liver function within hours of $\mathrm{BDL}$.

\section{HETEROGENEITY IN THE DIAGNOSIS OF COELIAC DISEASE IN PAEDIATRIC PATIENTS}

doi:10.1136/archdischild-2012-302724.0722

${ }^{1,2 \mathrm{D}}$ Croaker, ${ }^{3} \mathrm{~J}$ Swann, ${ }^{4} \mathrm{~L}$ Hallam, 1,2P Jenkins, ${ }^{5} \mathrm{C}$ Beardsley. ${ }^{1}$ Dept of Paediatrics and Child Health, The Canberra Hospital; '2Australian National University, Canberra, ACT, ${ }^{3}$ Wollongong University, Wollongong, NSW; ${ }^{4}$ Anatomical Pathology; ${ }^{5}$ Department of Surgery, The Canberra Hospital, Canberra, ACT, Australia

Background "Gluten intolerance" is commonly diagnosed and often confused in the public mind with coeliac disease. Authors in Western Australia recently demonstrated an approximately 5\% rate of coexistence of eosinophilic oesophagitis (EO) with villous atrophy. We asked whether our population was similar.

Methods We performed a retrospective chart review of all those with gastroscopy and small-bowel biopsy and a subsequent diagnosis of CD in children less than 16 years of age between 1 April 2003 and the 31st of June 2011.

Main results 239 gastroduodenoscopies were reviewed. Biopsy of both the oesophagus and duodenum was available in 231 patients. There were 124 patients positive for coeliac disease, 105 negative, and 10 indeterminate. 14 of 231 were positive for $\mathrm{EO}$, and 4 of the $126 \mathrm{CD}$ patients were also positive for $\mathrm{EO}$. Two of the four $\mathrm{CD}+\mathrm{EO}$ patients were rescoped during the time interval, and both were in remission for changes of $\mathrm{CD}$, although both still had changes of $\mathrm{EO}$ evident. There were $7 \mathrm{CD}$ patients reported with other forms of oesophageal inflammation.

Histologically of our four patients with EO and villous atrophy, at least three have potentially allergic changes instead of fullblown CD.

Conclusion There have been recent suggestions that serological and other tests may render the small bowel biopsy unnecessary in the management of $\mathrm{CD}$. We note that $\mathrm{EO}$ is associated with villous atrophy in 3 to $4 \%$ cases. Serology and symptomatology presenting as coeliac disease continues to warrant detailed investigation, including endoscopic work up.

\section{REVIEW OF TTG VERSUS SMALL INTESTINAL BIOPSY RESULTS; DO WE STILL NEED DUODENAL BIOPSY TO DIAGNOSE COELIAC DISEASE?}

doi:10.1136/archdischild-2012-302724.0723

TIY Hassan, S Quinn. Paediatrics, AMNCH, Dublin, Ireland

Aims To assess compare the results of tissue transglutaminase (tTg) with small intestinal biopsy results in children who had oesphageogastroduodenoscopy (OGD) to assess for coeliac disease (CD) at the National Children's Hospital, Tallaght, between January 2008 and December 2009.

Methods We reviewed the patients' records for all OGDs performed to assess for CD during the study period. Small intestinal biopsy results versus the $\mathrm{t} \mathrm{T}$ results were recorded.

Results 61 patients had an OGD performed during this period for assessment for CD (age 2-15 years). Three were excluded because no $\mathrm{tTg}$ was performed or recorded. Of these, 26 patients were males with male to female ratio of 0.8:1.

Fifty eight patients were included in the study, 32 had positive intestinal biopsy. 